\title{
An order-theoretic approach to involutive algebras
}

by

HERMANN RENDER (Duisburg)

Abstract. Let $A$ be a not necessarily associative *-algebra with a locally convex vector space topology. We investigate the order properties of the closed wedge $\bar{A}_{+} \cap \operatorname{Sym} A$ and obtain a description via positive functionals. Our main result is a new characterization for the a description via positive functionals. Our main result is a new characterization for the
antisymmetry of the wedge with various applications. For example, we prove that the continuity of the involution is necessary for the automatic continuity of positive functionals on certain algebras. Furthermore, we deduce that the closed wedge of a semisimple Waelbroeck algebra with a hermitian involution is a cone and unique in the sense that any other involution induces a non-antisymmetric closed wedge.

Introduction. Let $A$ be a not necessarily associative complex *-algebra. By $\operatorname{Sym} A$ we denote the real subspace of all selfadjoint elements of $A$. It is well known that the set

$$
A_{+}:=\left\{\sum_{i=1}^{N} a_{i}^{*} a_{i} ; N \in N, a_{i} \in A, i=1, \ldots, N\right\}
$$

is a wedge which induces a partial order $\leqslant$, i.e. a reflexive and transitive relation, where $a \leqslant b$ is defined by $b-a \in A_{+}$. There is a vast literature concerning specific order properties of $C^{*}$-algebras and, sometimes, Banach *algebras (see [5], [7], [10] and the references in [19]). The aim of this paper is a systematic study of order properties of complex involutive algebras.

In the first section we collect some basic facts on order properties of *-algebras. For example, each sequentially complete spectrally bounded LMC *-algebra is an order unit space. In the sequel our main tool is a separation theorem for a closed wedge and a point outside, so we consider not necessarily associative *-algebras endowed with a locally convex vector space topology. As a matter of fact, *-algebras are often pathological from the order-theoretic point of view, since in general the closed wedge $\bar{A}_{+}$is not a cone.

In the second section we give new characterizations of *-algebras having a closed cone $\bar{A}_{+}$, which rest on the simple observation that $\operatorname{Sym} A \cap i \operatorname{Sym} A$ $=\{0\}$. As a consequence we show that these algebras not only possess enough positive functionals but also a maximal quantity of positive func- 
tionals. Furthermore, this characterization yields an interesting result about the automatic continuity of positive functionals on a not necessarily associative *-algebra $A$ endowed with a Fréchet vector space topology: If $\bar{A}_{+} \cap \operatorname{Sym} A$ is antisymmetric then the continuity of the involution is necessary for the automatic continuity.

In the third section we consider a special class of commutative algebras, namely the Waelbroeck algebras. We obtain a surprisingly elegant description of the order wedge $\hat{A}_{+}$, the closure of the image of $A_{+}$under the Gelfand map. Then we show that the closed wedge of a semisimple Waelbroeck algebra with a hermitian involution is antisymmetric and unique in the sense that any other involution induces a closed wedge which is not antisymmetric. Finally, we determine all involutions on the disk algebra.

1. Preliminary results. We say that the wedge $A_{+}$generates $A$ if the complex linear span of $A_{+}$is the entire algebra $A$, i.e. $\operatorname{Lin}_{C} A_{+}=A$. The polarization formula

$$
\begin{aligned}
4 b a= & \left(a+b^{*}\right)^{*}\left(a+b^{*}\right)-\left(a-b^{*}\right)^{*}\left(a-b^{*}\right) \\
& +i\left(a+i b^{*}\right)^{*}\left(a+i b^{*}\right)-i\left(a-i b^{*}\right)^{*}\left(a-i b^{*}\right)
\end{aligned}
$$

shows that this is equivalent to the condition $A^{2}:=\operatorname{Lin}_{c}\{a b \mid a, b \in A\}=A$, which does not depend on the involution; of course the wedge $A_{+}$of a unital not necessarily associative ${ }^{*}$-algebra is always generating. Since the wedge $A_{+}$is contained in $\operatorname{Sym} A$ one may view $\operatorname{Sym} A$ as a real-linear ordered vector space with the order induced by $A_{+}$.

A linear operator $T: A \rightarrow B$ is called positive iff $T\left(A_{+}\right) \subset B_{+}$, or equivalently, $T\left(a^{*} a\right) \in B_{+}$for all $a \in A$. If the wedge $A_{+}$generates $A$ it is easy to see that a positive operator maps selfadjoint elements to selfadjoint ones, and that $T\left(a^{*}\right)=(T a)^{*}$. In particular, a positive functional, which can be considered as a positive operator with the ${ }^{*}$ algebra $C$ as range space, is always real on $\operatorname{Sym} A$ in the case that $A^{2}=A$. So there is a natural affine bijection between the set of all complex-linear positive operators on the entire algebra $A$ and the set of all real-linear positive operators on $\operatorname{Sym} A$, defined by restriction to $\operatorname{Sym} A$ resp. for a real-linear positive operator $L: \operatorname{Sym} A$ $\rightarrow \operatorname{Sym} A$ by the formula $T_{L}(u+i v):=L u+i L v$ with $u, v \in \operatorname{Sym} A$. If the algebra $A$ is endowed with a vector space topology we define the dual cone $A_{+}^{\prime}:=\{f \mid f: A \rightarrow C$ positive and continuous\}.

For a real locally convex vector space it is well known that one can separate a closed wedge and a point outside of it by a continuous functional $f: \operatorname{Sym} A \rightarrow \boldsymbol{R}$ (see e.g. [16, p. 58]). Since the wedge is positive-homogeneous and contains zero the functional $f$ or. $-f$ is positive relative to the closed wedge. Now the wedge $\bar{A}_{+} \cap \operatorname{Sym} A$ is a closed wedge in $\operatorname{Sym} A$ endowed with the relative topology. So we can separate each selfadjoint element outside of $\bar{A}_{+} \cap \operatorname{Sym} A$ by a real-linear continuous positive functional, which can be extended to a complex-linear positive functional on the entire algebra. If the continuity of the involution is assumed it is obvious that this extension is also continuous on $A$. But in some cases the continuity of the involution is not necessary to obtain the continuity of the extension. For example, every positive functional on a Banach *-algebra is automatically continuous. We circumvent this difficulty by dealing with the larger set $\widetilde{A}_{+}^{\prime}$ of all positive functionals on $A$ whose restrictions to $\operatorname{Sym} A$ are continuous. Our arguments prove the nontrival part of the following separation theorem.

1.1. Proposition. Let $A$ be a not necessarily associative complex *algebra with a locally convex vector space topology. Then an element $a \in A$ is contained in $\bar{A}_{+} \cap \operatorname{Sym} A$ if and only if $a=a^{*}$ and $f(a) \geqslant 0$ for all $f \in \tilde{A}_{+}^{\prime}$.

Note that $\bar{A}_{+}=\vec{A}_{+} \cap \operatorname{Sym} A$ if the involution is continuous. If $A$ is unital we define $\tilde{P}_{A}:=\left\{f \in \widetilde{A}_{+}^{\prime} ; f(1)=1\right\}$ and $P_{A}:=\left\{f \in A_{+}^{\prime} ; f(1)=1\right\}$. The Cauchy-Schwarz inequality shows that a positive functional $f$ with $f(1)=0$ is the zero functional. It follows that $\tilde{P}_{A}$ is a base of $\tilde{A}_{+}^{\prime}$. We denote the set of all extreme points of $\widetilde{P}_{A}$ by ext $\tilde{P}_{A}$.

1.2. Corollary. Let $A$ be a not necessarily associative unital *-algebra with a locally convex vector space topology. If $\tilde{P}_{A}$ is the weak star closure of the convex hull of its extreme points then $a \in A$ is contained in $\bar{A}_{+} \cap \operatorname{Sym} A$ iff a $=a^{*}$ and $f^{\prime}(a) \geqslant 0$ for all $f \in$ ext $\widetilde{P}_{A}$.

We call an element $e \in A$ of a not necessarily associative ${ }^{*}$-algebra $A$ an order unit if the complex linear span of the order interval $[0, e]:=\{a \in A$; $0 \leqslant a \leqslant e\}$ is the entire algebra, i.e. $\operatorname{Lin}_{c}[0, e]=A$. If $A$ is a unital associative ${ }^{*}$-algebra we denote the spectral radius by ||$_{\sigma}$. Assume that $A$ is spectrally bounded, i.e. every element has a bounded spectrum, and let $a \in A$ be such that we can define for all $\varepsilon>0$ a selfadjoint square root

$$
b:=\sqrt{1-\frac{a^{*} a}{\left|a^{*} a\right|_{\sigma}+\varepsilon}}
$$

Then we have $a^{*} a \in\left[0,\left(\left|a^{*} a\right|_{\sigma}+\varepsilon\right) \cdot 1\right]$, since $a^{*} a=\left(\left|a^{*} a\right|_{\sigma}+\varepsilon\right) \cdot\left(1-b^{2}\right)$ $\leqslant\left(\left|a^{*} a\right|_{\sigma}+8\right) \cdot 1$. As $A$ is unital each element can be decomposed into a linear combination of positive elements and we obtain $\operatorname{Lin}[0,1]=A$, if there are enough selfadjoint square roots. In the case of a unital sequentially complete LMC algebra with a continuous involution there exist such square roots for elements with bounded spectrum (cf. [9, p. 15]). An algebra with a locally convex vector space topology is called a $L M C$ algebra iff there exists a family $\left(p_{\alpha}\right)_{\alpha, I}$ of submultiplicative seminorms $p_{\alpha}$ generating the topology of $A$. Such a family is called a defining family (cf. [9, p. 3]).

In the case of a Banach *algebra the lemma of Ford is available (see e.g. [1]). So we have proved the following. 
1.3. Proposmion. Let $A$ be a unital sequentially complete spectrally bounded LMC algebra with a continuous involution or a unital Banach *-algebra. Then the unit element is an order unit and $0 \leqslant a^{*} a \leqslant\left|a^{*} a\right|_{\text {s }}+\varepsilon$ for each $\varepsilon>0$.

Of course, there are algebras having the unit element as order unit without being complete.

Proposition 1.1 characterizes the closed wedge $\bar{A}_{+} \cap \operatorname{Sym} A$. The proof is essentially based on methods of convex analysis. Now we want to give a characterization for the property that the unit element is an order unit for $\bar{A}_{+} \cap \operatorname{Sym} A$.

First we need the following definition. Let $S$ be a set of linear functionals on $A$. Then $S$ induces a "seminorm" $|\cdot|_{s}$ with values in $[0, \infty]$ defined by $|a|_{S}:=\sup ,|f(a)| ; f \in S_{1}$. Clearly, the function $|\cdot|_{S}$ is real-valued iff $S$ is $w^{*}$-bounded. Note that $|a|_{P_{A}}$ and $|a|_{\tilde{P}_{A}}$ are defined for all $a \in A$.

1.4. Proposition. Let $A$ be a not necessarily associative unital *-algebra with a locally convex vector space topology. Then the unit element is an order unit for the wedge $\ddot{A}_{+} \cap \operatorname{Sym} A$ iff the set $\tilde{P}_{A}$ is $w^{*}$-bounded. In that case $-|u|_{\tilde{P}_{A}} \leqslant u \leqslant|u|_{\bar{P}_{A}}$ relative to $A_{+} \cap \operatorname{Sym} A$ for all $u \in \operatorname{Sym} A$.

Proof. For every $u \in[0,1]$ we obtain $0 \leqslant f(u) \leqslant f(1)=1$ for all $f \in \tilde{P}_{A}$. If 1 is an order unit, each element is a linear combination of such elements, which shows $|a|_{\tilde{P}_{A}}<\infty$, i.e. $\tilde{P}_{A}$ is $w^{*}$-bounded.

Let $u$ be selfadjoint and assume that $\tilde{P}_{A}$ is $w^{*}$-bounded, i.e. $|u|_{\tilde{P}_{A}}<\infty$. From the delinition of $|u|_{\tilde{P}_{A}}-u$ we see that $f\left(|u|_{\tilde{P}_{A}}-u\right) \geqslant 0$ for every $f \in \tilde{P}_{A}$ and by Proposition 1.1 the positivity of $|u|_{\tilde{P}_{A}}-u$ is evident. Replacing $u$ by $-u$ we obtain the desired inequality $-|u|_{\tilde{P}_{A}} \leqslant u \leqslant|u|_{\tilde{P}_{A}}$. Thus the unit element is an order unit.

The radical $\operatorname{rad} A$ of an associative algebra can be defined as the intersection of all maximal modular left ideals of $A$. The algebra is called semisimple if the radical is trivial. It is well known that $a \in A$ is in the radical iff $|a b|_{\sigma}=0$ for all $b \in A$ (cf. [1, pp. 124-126]).

1.5. Proposition. Let $A$ be a unital involutive $L M C$ algebra. Then each $f \in \tilde{P}_{A}$ vanishes on the radical of $A$. We have $P_{A}=\tilde{P}_{A}$ for all commutative algebras with continuous spectral radius and for all Banach *algebras.

Proof. Let $\left(p_{\alpha}\right)_{\alpha \notin I}$ be a defining family of submultiplicative seminorms and let $f \in \tilde{P}_{A}$. As $f$ is continuous on $\operatorname{Sym} A$ there exist a constant $c>0$ and $\alpha \in I$ with $|f(u)| \leqslant c p_{\alpha}(u)$ for all $u \in \operatorname{Sym} A$. Since $f$ is positive and unital, the Cauchy-Schwarz inequality shows that $|f(a)|^{2^{n+1}} \leqslant f\left(\left(a^{*} a\right)^{2^{n}}\right)$ for all $n \in N$ and therefore

$$
|f(a)|^{2} \leqslant \lim _{n \rightarrow \infty} c^{1 / 2^{n}} \lim _{n \rightarrow \infty}\left(p_{\alpha}\left(\left(a^{*} a\right)^{2^{n}}\right)\right)^{1 / 2^{n}} \leqslant\left|a^{*} a\right|_{\sigma}
$$

For the last inequality see $[1, \mathrm{p} .48]$. If $a$ is in the radical we have $\left|a^{*} a\right|_{\sigma}=0$, and this proves the first statement. The second statement is an easy consequence of $\left|a^{*} a\right|_{\sigma} \leqslant|a|_{\sigma}^{2}$ and the continuity of the spectral radius and, in the case of a Banach *-algebra, of the automatic continuity of positive functionals (see e.g. [1, p. 198]).

2. When is the closed wedge a cone? A partial order $\leqslant$ is called antisymmetric iff $0 \leqslant a \leqslant 0$ implies $a=0$, or equivalently, iff the set $A_{+}$of all positive elements is a cone, i.e. a wedge with the relation $A_{+} \cap-A_{+}=\{0\}$. In the sequel we will discuss criteria for the antisymmetry of the wedge $\bar{A}_{+} \cap \operatorname{Sym} A$. Our main result is

2.1. Theorem. Let $A$ be a not necessarily associative *algebra with $A^{2}$ $=A$ endowed with a locally convex vector space topology. Then the following statements are equivalent:

(a) $\bar{A}_{+} \cap \mathrm{Sym} A$ is antisymmetric.

(b) $f(a) \geqslant 0$ for all $f \in \widetilde{A}_{+}^{\prime}$ implies $a \in \bar{A}_{+} \cap \operatorname{Sym} A$.

(c) $f(a) \in R$ for all $f \in \tilde{A}_{+}^{\prime}$ implies $a \in \operatorname{Sym} A$.

(d) For all $a \in A \backslash\{0\}$ there exists $f \in \tilde{A}_{+}^{\prime}$ with $f(a) \neq 0$.

Proof. We show the implication (a) $\Rightarrow(\mathrm{b})$. Let $a \in A$ and suppose $f(a)$ $\geqslant 0$ for all $f \in \tilde{A}_{+}^{\prime}$. By Proposition 1.1 it is sufficient to show the selfadjointness of $a$. But for $a=u+i v$ with $u, v \in \operatorname{Sym} A$ we have $f(v)=0$ for all $f \in \tilde{A}_{+}^{\prime}$, since each positive functional is real on $\operatorname{Sym} A$ and $f(a) \geqslant 0$. Hence we conclude by Proposition 1.1 that $v \in \vec{A}_{+} \cap-\vec{A}_{+} \cap \operatorname{Sym} A=\{0\}$.

Now let us assume (b). We consider $v:=a^{*}-a$ and have to show $v=0$ if $f(a) \in \boldsymbol{R}$ for all $f \in \tilde{A}_{+}^{\prime}$. But since $f(v)=f\left(a^{*}-a\right)=\overline{f(a)}-f(a)=0$, assumption (b) shows that $v$ and $i v$ are contained in $\operatorname{Sym} A$, which gives $v=0$.

For the implication (c) $\Rightarrow$ (d) let $a=u+i v$ with $u, v \in \operatorname{Sym} A$. Assume that $f(a)=0$ for all $f \in \tilde{A}_{+}^{\prime}$. Since $f(u), f(v)$ are real we have $f(u)=f(v)=0$. Assumption (c) shows that $i u$, $i v$ are selfadjoint and thus $u=v=0$. The last implication is trivial.

The theorem shows that $\bar{A}_{+} \cap \operatorname{Sym} A$ is antisymmetric iff there are enough positive continuous (relative to $\operatorname{Sym} A$ ) functionals to separate the points of $A$. But we will also show that the antisymmetry furnishes even a maximal quantity of positive functionals.

In $[8, p .68]$ an involution is called essential iff for all $a \in A \backslash\{0\}$ there exists a positive functional $f$ with $f\left(a^{*} a\right) \neq 0$. Let us consider the condition

(e) For all $a \in A \backslash\{0\}$ there exists $f \in \tilde{A}_{+}^{\prime}$ with $f\left(a^{*} a\right) \neq 0$.

If $A$ is unital the Cauchy-Schwarz inequality shows that (d) implies (e). In particular, the antisymmetry implies that the involution is proper, i.e. $a^{*} a \neq 0$ for all $a \in A \backslash\{0\}$ (cf. [4, p. 420]). On the other hand, (e) implies (d) if 
the multiplication is associative and separately continuous: if $f(a)=0$ for all $f \in \tilde{A}_{+}^{\prime}$ consider the positive functional $\varphi(a):=f\left((\lambda+b) a\left(\bar{\lambda}+b^{*}\right)\right)$ with $a, b \in A$ and $\lambda \in C$. Then it is easy to see that $f\left(a^{*} a\right)=0$ for all $f \in \tilde{A}_{+}^{\prime}$ (see e.g. [11, pp. 261-262]).

If $A$ is a unital Banach *-algebra then every positive functional is automatically continuous. The Gelfand-Naimark pseudonorm $m_{A}$, defined by $m_{A}(a):=\sqrt{\left|a^{*} a\right|_{P_{A}}}$, is a $C^{*}$-seminorm. The ${ }^{*}$-radical can be defined as the kernel of $m_{A}$ (cf. [14, Theorems 4.2 and 4.4]). Thus the closed wedge is

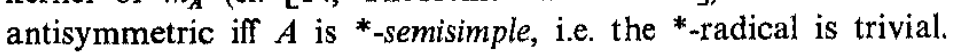

2.2. Corollary. Let $A$ be a not necessarily associative *algebra with $A^{2}$ $=A$ and a Fréchet space. Assume that $\bar{A}_{+} \cap \operatorname{Sym} A$ is antisymmetric. Then the continuity of the involution is a necessary condition for the automatic continuity of positive functionals.

A locally convex vector space is called a Fréchet space if its topology is induced by a complete translation invariant metric. Note that there is no assumption of continuity of the multiplication in our theorem.

For more general *-algebras (than Banach *-algebras) the continuity of the involution has been required to prove the continuity of positive functionals (cf. the survey [6]). Our theorem justifies this assumption. In the case of a Banach *-algebra $A$ one has first to show that the involution is continuous on $A / \mathrm{rad} A$ in order to prove the automatic continuity of positive functionals.

As a consequence of Corollary 2.2 we infer for a Banach *-algebra $A$ that $\bar{A}_{+} \cap \operatorname{Sym} A$ is antisymmetric iff $\bar{A}_{+}$is antisymmetric, since every positive functional is automatically continuous. Now let us prove the corollary.

Proof. The automatic continuity implies $\tilde{A}_{+}^{\prime}=A_{+}^{\prime}$. If $\bar{A}_{+} \cap \operatorname{Sym} A$ is antisymmetric Theorem 2.1(c) tells us that

$$
\operatorname{Sym} A=\bigcap_{S \in \tilde{A}_{+}^{\prime}} f^{-1}(R) .
$$

Thus the property $\tilde{A}_{+}^{\prime}=A_{+}^{\prime}$ implies that $\operatorname{Sym} A$ is closed in $A$. Since $A$ is a Fréchet space we can apply the closed graph theorem to prove the continuity of the involution (see e.g. [16, p. 50]). Let $\left(a_{n}\right)_{n \in N}$ be a sequence in $A$ such that $\left(a_{n}\right)_{n \in N}$ converges to $a \in A$ and $\left(a_{n}^{*}\right)_{n \in N}$ converges to $b \in A$. We have to show that $a^{*}=b$. Clearly $a_{n}+a_{n}^{*} \rightarrow a+b$ and $i\left(a_{n}-a_{n}^{*}\right) \rightarrow i(a-b)$. Since Sym $A$ is closed we can conclude that $a+b$ and $i(a-b)$ are selfadjoint, thus $a+b=a^{*}+b^{*}$ and $a-b=b^{*}-a^{*}$, which yields $a^{*}=b$.

Let $A$ be a not necessarily associative *-algebra with a locally convex vector space topology and let $x$ be another involution. We denote the set of all functionals which are positive relative to $x$ and continuous on the selfadjoint part of $A$ by $\tilde{A}_{+}^{\prime}(\times)$. Now we consider the set

$$
\mathscr{P}:=\left\{\tilde{A}_{+}^{\prime}(x) \mid x \text { is an involution on } A_{\}}\right.
$$

and define an ordering on $\mathscr{P}$ by set inclusion.
2.3. Corollary. Let $A$ be a not necessarily associative *algebra with $A^{2}=A$ endowed with a locally convex vector space topology. If the closed wedge $\bar{A}_{+} \cap \operatorname{Sym} A$ is antisymmetric then $\widetilde{A}_{+}^{\prime}(*)$ is a maximal element in $: \mathcal{P}$.

Proof. Let $\times$ be another involution with $\tilde{A}_{+}^{\prime}(*) \subset \tilde{A}_{+}^{\prime}(\times)$. For $a=a^{+}$ we have $f(a) \in \boldsymbol{R}$ for all $f \in \tilde{A}_{+}^{\prime}(x)$, in particular for each $f \in \tilde{A}_{+}^{\prime}(*)$. By Theorem 2.1 we conclude $a=a^{*}$. But now it is easy to see that the involutions $*$ and $\times$ coincide.

2.4. Remark. By Corollary 1.2 it is easy to see that one may replace $\tilde{A}_{+}^{\prime}$ by the set of all extreme points of $\tilde{P}_{A}$ in Theorem 2.1, Corollary 2.3 and (1) if $\tilde{P}_{A}$ is the $w^{*}$-closed convex hull of its extreme points.

An involution of an associative algebra is called hermitian if the spectrum of every selfadjoint element is real. Now let $A$ be a Banach algebra with a hermitian involution. Then the radical and the ${ }^{*}$-radical coincide (see [14, Theorem 6.6]). Thus, if $A$ is semisimple, the wedge $\bar{A}_{+}$is antisymmetric. We generalize this result to complete LMC algebras with a continuous involution.

2.5. THEOREM. Let $A$ be a complete semisimple LMC algebra with a continuous hermitian involution. Then the closed wedge $\bar{A}_{+}$is antisymmetric.

Proof. Without loss of generality we can assume that $A$ possesses a unit. Let $\left(p_{\alpha}\right)_{\alpha \in I}$ be a defining family of submultiplicative seminorms. The completion of the normed space $A / p_{\alpha}^{-1}\{0\}$ is denoted by $A_{\alpha}$ and the image of $a \in A$ in $A_{\alpha}$ is denoted by $a_{\alpha}$. It is well known that $A$ is isomorphic to the projective limit of the Banach *-algebras $A_{\alpha}$ (cf. [9, p. 12]). In particular, the involution of every $A_{\alpha}$ is hermitian. By $P_{\alpha}$ we denote the set of all unital positive functionals which are continuous relative to the seminorm $p_{\alpha}$. Clearly then $P_{A}=\bigcup_{\alpha \in I} P_{\alpha}$. It is easy to see that $P_{\alpha}$ and $P_{A_{\alpha}}$ are affinely isomorphic (cf. [2]). Let $a \in \bar{A}_{+} \cap-\bar{A}_{+}$, i.e. $f(a)=0$ for all $f \in P_{A}$. This implies $g\left(a_{\alpha}\right)=0$ for all $g \in P_{A_{\alpha}}$. But then $a_{\alpha}$ is in the radical of $A_{\alpha}$, since $A_{\alpha}$ is a Banach *-algebra. So $a_{\alpha} \in \operatorname{rad} A_{\alpha}$ for all $\alpha \in I$, which yields $a \in \operatorname{rad} A$. Since $A$ is semisimple we obtain $a=0$, which completes the proof.

There exist Banach ${ }^{*}$-algebras with a closed cone $\bar{A}_{+}$but without a hermitian involution, e.g. the disk algebra. But if we require additional structures on the Banach *-algebra these notions coincide. The equivalence of statements (a) and (b) of the following theorem is due to Civin and Yood [4].

2.6. Theorem. Let $A$ be a commutative regular semisimple Banach *algebra. Then the following statements are equivalent:

(a) $*$ is a hermitian involution.

(b) The involution * is proper, i.e. $a^{*} a \neq 0$ for $a \in A \backslash\{0\}$.

(c) $A_{+}$is antisymmetric.

(d) $\bar{A}_{+}$is antisymmetric. 
Proof. Theorem 2.5 gives (a) $\Rightarrow$ (d), and $(d) \Rightarrow(c)$ is trivial. We now prove (c) $\Rightarrow$ (b). Let $a=u+i v(u, v \in \operatorname{Sym} A)$. The condition $a^{*} a=u^{2}+v^{2}=0$ implies that the positive element $u^{2}$ is also negative and the antisymmetry of $A$, gives $u^{2}=0$. Since $A$ is semisimple we can conclude $u=0$ and sinilarly $v$ $=0$. For the implication (b) $\Rightarrow$ (a) we refer to [4].

3. Involutions on Waelbroeck algebras. A Waelbroeck algebra is a complex commutative locally convex continuous inverse algebra. The importance of Waelbroeck algebras stems from the fact that the so-called Gelfand theory carries over to this larger class of topological algebras. By a result of $\mathrm{P}$. Turpin [20] the Waelbroeck algebras are exactly the commutative LMC $Q-$ algebras. The set of all nontrivial multiplicative functionals coincides with the set of all nontrivial multiplicative continuous functionals denoted by $\Delta_{A}$. The Gelfand transformation $G_{A}$ maps $a \in A$ to the continuous function $\hat{a} \in C\left(\Delta_{A}\right)$ defined by $\hat{a}(h):=h(a)\left(h \in \Delta_{A}\right)$. The spectral radius is a continuous submultiplicative seminorm and by Proposition 1.5 we obtain $\tilde{P}_{A}=P_{A}$. An involution * on a Waelbroeck algebra $A$ induces an involution on $\hat{A}$ (the image of the Gelfand map) via $\hat{a}^{*}:=\left(a^{*}\right)$

We will give a description of the ||$_{\sigma}$-closed wedge $\overline{\hat{A}}_{+}$. The set $P_{\hat{A}}$ of all positive continuous (relative to the spectral radius) unital functionals on $\hat{A}$ can be identified with $P_{A}$, since the map $g \rightarrow g \circ G_{A}$ is affinely bijective (for the surjectivity cf. Proposition 1.5 ). $\hat{A}$ is a ${ }^{*}$-algebra with the norm ||$_{\sigma}$ so we conclude by a result of R. S. Bucy and G. Maltese in [3] that

$$
\operatorname{ext} P_{\hat{A}}=P_{\hat{A}} \cap \Delta_{\hat{A}}, \quad P_{\hat{A}}=\overline{\text { conext } P_{\hat{A}}}{ }^{* *} \text {. }
$$

So we can identify ext $P_{\hat{A}}$ and $P_{A} \cap \Delta_{A}$. An application of Corollary 1.2 and Theorem 2.1 gives the following result.

3.1. Corollary. Let $A$ be a unital involutive Waelbroeck algebra. Then

$$
\hat{a} \in \overline{\hat{A}}_{+} \Leftrightarrow \hat{a}^{*}=\hat{a} \text { and } \hat{a}(s) \geqslant 0 \text { for all } s \in P_{A} \cap \Delta_{A} .
$$

If in addition the closed wedge $\overline{\hat{A}}_{+}$is antisymmetric then

$$
\hat{a} \in \bar{A}_{+} \Leftrightarrow \hat{a}(s) \geqslant 0 \text { for all } s \in P_{\boldsymbol{A}} \cap \Delta_{A_{A}} \text {. }
$$

It is well known (see e.g. [4]) that an involution on a Waelbroeck algebra can be described by a homeomorphism $\varphi$ of the structure space $A_{A}$ which has period two, i.e. $\varphi \circ \varphi=\mathrm{id}_{\Delta_{A}}$. This homeomorphism is characterized by the relation $\varphi(h)=\bar{h} \circ *$ with $\bar{h} \in \Delta_{A}$. Then $\hat{a}^{*}=\hat{a} \circ \varphi$ for each $a \in A$, and using the relation ext $P_{A}=P_{A} \cap \Delta_{A}$, one obtains an easy proof that the extreme points of $P_{A}$ are exactly the fixed points of $\varphi$. If the structure space $\Delta_{A}$ is homeomorphic to a compact convex subset of a locally convex vector space an application of a fixed point theorem (cf. [14, p. 120]) proves the existence of a positive continuous functional.

For a Waelbroeck algebra we have $\sigma(a)=\hat{a}\left(\Delta_{A}\right)$ and $\hat{a}^{*}=\tilde{\hat{a}} \circ \varphi$. Then it is easy to see that the involution $*$ is hermitian if and only if $\varphi$ is the identity. This shows that the involution is hermitian iff ext $P_{A}=\Delta_{A}$, since the extreme points are exactly the fixed points of $\varphi$. In particular, there is at most one hermitian involution for a semisimple Waelbroeck algebra. In the noncommutative case Civin and Yood have shown in [4] that on a Banach *-algebra there are denumerably infinitely many distinct hermitian involutions if there, exists at least one such involution.

Now we are able to give an interesting application of Corollary 2.3: Let $A$ be a unital semisimple Waelbroeck algebra with a hermitian involution $*$. Let $\times$ be another involution and assume that the closed wedge $\bar{A}_{+}$relative to $x$ is antisymmetric. Corollary 2.3 and Remark 2.4 show that ext $P_{A}(x)$ is a maximal set and thus the relation ext $P_{A}(x)=P_{A}(x) \cap \Delta_{A} \subset \Delta_{A}$ $=$ ext $P_{A}(*)$ yields ext $P_{A}(\times)=\Delta_{A}$. Since $\bar{A}_{+}(\times)$is antisymmetric and the algebra is semisimple, this implies $x=*$. We have proved the following result:

3.2. Corollary. Let $A$ be a unital semisimple Waelbroeck algebra with a hermitian involution. Then the closed wedge of no other involution is antisymmetric.

Examples. (a) We determine all involutions on the disk algebra. We identify its structure space with the unit disk $D$ in the complex plane. The involution induces a homeomorphism $\varphi: D \rightarrow D$ of period two with $a^{*}(z)$ $=\bar{a} \circ \varphi(z)(z \in D)$. Thus $\bar{\varphi}$ is an element of the disk algebra since the identity function is in $A$. Simple arguments show that $\bar{\varphi}$ is an automorphism of $D$, hence has the general form

$$
\frac{a z+b}{\bar{b} z+\bar{a}} \text { with }\left(\frac{a}{b} \frac{b}{\bar{a}}\right) \in \mathrm{SL}_{2}(C),
$$

and that $\varphi \circ \varphi=\mathrm{id}_{D}$ iff $b$ is purely imaginary. So we have

$$
\varphi(z)=\frac{\overline{a z}-i \beta}{i \beta \bar{z}+a}
$$

with $a \in C, \beta \in R$ and $|a|^{2}-\beta^{2}=1$. The fixed points of $\varphi$, i.e. the extreme points of $P_{A}(\varphi)$, are the solutions in $D$ of the equation $i \beta \bar{z} z+a z-\overline{a z}+i \beta=0$, which for $\beta=0$ describes a straight line through zero and for $\beta \neq 0$ a circle with center $\vec{a} /(i \beta)$ and radius $1 /|\beta|$ which intersects the unit disk. One may view $\varphi$ as an antiholomorphic reflection of the orthogonal circle consisting of the fixed points of $\varphi$. In particular, each involution on $A$ has an antisymmetric wedge $\bar{A}_{+}$

(b) We give an example of a Banach *-algebra $A$ where $\bar{A}_{+}$is a cone different from $A_{+}$. Let $A$ be the set of all functions of the disk algebra whose derivatives may be extended to continuous functions on the closed unit disk. $A$ is a Banach algebra with the norm $\|a\|:=\|a\|_{\infty}+\left\|a^{\prime}\right\|_{\infty}$, where $a^{\prime}$ denotes 
the derivative of $a$, and with the involution $a^{*}(z)=\bar{a}(\bar{z})$. The extreme points of $P_{A}$ can be identified with $[-1,1]$, the set of all fixed points of $z \rightarrow \bar{z}$. By Corollary 3.1 the function $a$ defined by $a(z):=1-z$ is contained in the cone $\bar{A}_{+}$, but it is not in $A_{+}$: Let $a(z)=1-z=\sum_{i=1}^{N} a_{i}^{*}(z) a_{i}(z)$ be a combination of positive elements. Differentiating both sides and using the continuity of $a^{\prime}$ on $D$ we obtain $a^{\prime}(1)=-1=0$, a contradiction.

\section{References}

[1] F. F. Bonsall and J. Duncan, Complete Normed Algebras, Springer, Berlin 1973.

[2] R. M. Brooks, On locally m-convex algebras, Pacific J. Math. 23 (1967), 5-23.

[3] R. S. Bucy and G. Malt ese, A representation theorem for positive functionals on involution algebras, Math. Ann. 162 (1966), 365-367.

[4] P. Civin and B. Yood, Involutions on Banach algebras, Pacilic J. Math. 9 (1959), 415-436.

[5] P. C. Curtis, Order and commutativity in Banach algebras, Proc. Amer. Math. Soc. 9 (1958), 643-646.

[6] P. G. Dix on, Automatic continuity of positive functionals on topological involution algebras, Bull. Austral. Math. Soc. 23 (1981), 265-281.

[7] R. S. Doran and V. A. Belfi, Characterizations of C*-Algebras, Dekker, 1986.

[8] 1. M. Gelfand, D. A. Raikow und G. E. Schilow, Kommutative normierte Algebren, Deutscher Verlag der Wissensch., Berlin 1964.

[9] T. Husain, Multiplicutive Functionals of Topological Algebras, Res. Notes in Math. 85 Pitman, Boston 1983.

[10] J. L. Kelley and R. L. Vaught, The positive cone in Banach algebras, Trans. Amer Math. Soc. 74 (1953), 44-55

[11] M. A. Naimark, Normed Algebras, 3rd ed., Wolters-Noordhoff, Groningen 1972.

[12] T. W. Palmer, The Gelfand-Naimark pseudo-norm on Banach *-algebraș, J. London Math Soc. (2) 3 (1971), 89-96.

[13] M. Pa nnenberg, Koroukin approximation in Waelbroeck algebras, Math. Ann. 274 (1986) 423-427.

[14] V. Pt ák, Banach algebras with involution, Manuscripta Math. 6 (1972), 245-290.

[15] H. Render, A characterization of $C^{*}$-algebras via positive operators, Arch. Math. (Basel) 50 (1988), to appear.

[16] W. Rudin, Functional Analysis, McGraw-Hill, New York 1973.

[17] H. Schaefer, Halbgeordnete lokal-konvexe Vektorräume III, Math. Ann. 141 (1966), 113 142.

[18] -, Topological Vector Spaces, Graduate Texts in Math. 3, Springer, Berlin 1971.

[19] D. M. Topping, Vector lattices of selfadjoint operators, Trans. Amer. Math. Soc. 115 (1965), 14-30.

[20] L. Waelbroeck, Topological Vector Spaces and Algebras, Lecture Notes in Math. 230, Springer, Berlin 1970.

UNIVERSITÄT-GESAMTHOCHSCHULE DUISBURO

Lotharstr. 65, D-4100 Duisburg, F.R.G.

\section{On the integrability and $L^{1}$-convergence of sine series *}

by

FERENC MÓRICZ (Szeged)

Abstract. We study sine series $(*) \sum_{k=1}^{\infty} a_{k} \sin k x$ with coefficients belonging to one or two of the classes $\tilde{C}, \tilde{B} V$, and $\tilde{V}_{p}$ introduced in this paper. Among other things, we prove that if $\left\{a_{k}\right\} \in \tilde{C} \cap \tilde{B} V$, then (*) is the Fourier series of some function $f \in L^{1}(0, \pi)$. Furthermore, if $\left\{a_{1}\right\} \in \tilde{C} \cap \tilde{B} V$ or $\{a\} \in \tilde{V}_{n}$ and $f \in L D(0, \pi)$, then the condition $(x), a_{n} \log n \rightarrow 0$ is necess suffeient for the $L(0, \pi)$ convergence of the partalion $(* *)$ has been known so far only in the case of cosine series, Our results generalize those obtained by Telyakovskii [9] for sine series, while our new classes are the counterparts of those introduced by Garrett and Stanojevic [5] as well as by Bojanic and Stanojevic [2] for cosine series.

1. Introduction. We will study the sine series

$$
\sum_{k=1}^{\infty} a_{k} \sin k x
$$

where $\left\{a_{k}\right\}$ is a sequence of real numbers in the class $\tilde{B} V$ defined as follows

Definition 1. A null sequence $\left\{a_{k}\right\}$ belongs to $\tilde{B} V$ if

$$
\sum_{k=1}^{\infty} k\left|\Delta b_{k}\right|<\infty
$$

where

$$
b_{k}:=a_{k} / k, \quad \Delta b_{k}:=b_{k}-b_{k+1} \quad(k=1,2, \ldots) .
$$

We do not require any monotonicity of the sequences $\left\{a_{k}\right\}$ and $\left\{b_{k}\right\}$.

Following an idea of Kano [6], we represent the partial sums $s_{n}(x)$ of series (1.1) in the form

$$
s_{n}(x)=\sum_{k=1}^{n} a_{k} \sin k x=-\sum_{k=1}^{n} b_{k}(\cos k x)^{\prime}
$$

1980 Mathematics Subject Classification: Primary 42A20, 42A32

Key words and phrases: integrability of sine series, $L^{1}$-convergence of Fourier series, modified sine sums.

* This research was completed while the author was a visiting professor at Syracuse University, Syracuse, New York, U.S.A., during the academic year 1986/87. 